\title{
Space-time properties of the higher twist amplitudes
}

\author{
Wei Zhu ${ }^{1,2}$, Hongwei Xiong ${ }^{2}$ and Jianhong Ruan ${ }^{2,3}$ \\ ${ }^{1}$ CCAST(World Laboratory), P.O.Box 8730, Beijing 100080, P.R. China \\ ${ }^{2}$ Department of Physics, East China Normal University, Shanghai 200062, P.R. China \\ ${ }^{3}$ Institute of High Energy Physics, Chinese Academy of Sciences, Beijing 100039, P.R. China
}

\begin{abstract}
A consistent and intuitive description of the twist-4 corrections to the hadron structure functions is presented in a QCD-improved parton model using timeordered perturbative theory, where the collinear singularities are naturally eliminated. We identify the special propagators with the backward propagators of partons in time order.
\end{abstract}

PACS numbers: 12.38.Bx, 13.85.Qk, 11.15.Bt 


\section{Introduction}

The twist- 4 or inverse-power $1 / Q^{2}$ corrections to scaling in deep inelastic scattering (DIS) processes are an important subject for a precision test of QCD and the analyses of deep inelastic scattering (DIS) data [1-4]. Jaffe and Soldate (JS) used OPE method to relate the inverse-power corrections to the target matrix elements of local operators [2]. On the other hand, the QCD-improved parton model can provide a simple and intuitive description of scaling phenomena as a compensate method for the OPE technique. In this aspect, Jaffe [4] recasted the results of work [2] in parton model. Ellis, Furmanski, Petronzio [3] (EFP) and Qiu [4] try to construct a complete QCD-improved parton model including the inverse-power short-distance corrections based on the manipulation of stand Feynman diagrams. Where EFP's diagram-approach needs a special complicate procedure to pick up the short-distance contributions. Qiu advanced the above technique and proposed that a set of special propagators can isolate the short-distance effect in Feynman diagrams [4]. However, the dynamic origin of the special propagators and collinear safeness of the theory are not yet clear. Obviously, we still lack a consistent QCD-improved parton model to analyze the inverse-power corrections in DIS processes.

In this work, we try to realize the twist-4 short-distance corrections to the hadron structure functions using time ordered perturbative theory (TOPT). We find that the collinear singularity can be naturally eliminated if we generalize TOPT to the collinear limit. In this framework, the twist-4 short-distance corrections to the hadron structure functions will present an intuitive space-time picture. In particular, we identify Qiu's special propagators [4] with the backward propagators of partons in time order. Our new method provides a consistent way to study higher-twist processes in a QCD-improved 
parton model.

The outline of the paper is as follows. In Sec. 2, we shall generalize TOPT to the collinear limit. We find that the collinear and infrared singularities in amplitude will transfer to the interaction-time from the momentum-space in TOPT and they can be natural eliminated. Using this TOPT-framework, we shall give a set of calculating rules, which pick up the inverse-power short-distance corrections to the hadron structure functions in Sec. 3. We shall discuss the factorization scheme in Sec. 4. A summary is given in the last section.

\section{Collinear safeness}

As we know, TOPT is a natural way for studying space-time properties of the twist-2 DGLAP evolution equation [5]. In principle, a Feynman diagram can be decomposed to all possible time-ordered graphs in TOPT. However, in the leading-twist processes many TOPT amplitudes, which contain the backward-propagating partons, are suppressed in the infinite momentum frame (see Fig. 1). The reason is straightforward. The cross section of DIS is generally a function of the longitudinal momenta, $x_{i} P$ and transverse momenta, $l_{\perp}^{2}$, of partons. The terms with $x_{i} P$ are canceled in Fig. 1a due to the conservation of longitudinal momenta at every vertex and the final results depend only on $l_{\perp}^{-2}$ and give $\log Q^{2}$-corrections. Any backward-propagating parton in the splitting vertex will violate the balance among $x_{i} P$ in the energy terms and lead to $d l_{\perp}^{2} / P^{2} \rightarrow 0$. Thus, the backward-propagating partons are suppressed and the scattering amplitudes only contain the forward-propagating partons in the leading twist processes. In this work we find that the twist-4 amplitudes also expose the simple TOPT-form but the contributions of backward moving partons are no longer neglected. 
The higher twist corrections to structure functions are separated in the collinear expansion, where all transverse momenta of partons are taken as zero [4]. Therefore, we first generalize the TOPT-amplitude to the collinear limit. As an example, we consider the process $p_{1}+p_{2} \rightarrow p_{1}^{\prime}+p_{2}^{\prime}$, in which the parton momenta are parametrised as

$p_{1}:\left(p_{10}, l_{\perp}, p_{1 z}\right), p_{2}:\left(p_{20},-l_{\perp}, p_{2 z}\right), p_{1}^{\prime}:\left(p_{10}^{\prime}, l_{\perp}, p_{1 z}^{\prime}\right), P_{2}^{\prime}:\left(p_{10}^{\prime},-l_{\perp}, p_{2 z}^{\prime}\right), k:\left(k_{0}, 0_{\perp}, k_{z}\right)$

where the transverse momenta $l_{\perp}$ will be set to zero. We emphasize that our following results are independent of the parameters (1). We choose a coordinate frame such that

$$
\begin{gathered}
p^{\mu}=p \bar{n}^{\mu} \\
q^{\mu}=-x_{B} p \bar{n}^{\mu}+\frac{Q^{2}}{2 x_{B} p} n^{\mu} .
\end{gathered}
$$

The contributions of the Feynman propagator of fermion (quark or antiquark) with the momentum $k$ to the amplitude are

$$
\begin{gathered}
\int d^{4} k S(k)=\int \frac{d k_{0} d^{3} \vec{k}}{(2 \pi)^{4}} \frac{\gamma \cdot k}{k_{0}^{2}-\omega^{2}+i \epsilon}(2 \pi)^{4} \delta^{4}\left(p_{1}+p_{2}-k\right)(2 \pi)^{4} \delta^{4}\left(k-p_{1}^{\prime}-p_{2}^{\prime}\right) \\
=\frac{i}{2 \omega}\left\{\int_{-\infty}^{\infty} d t^{\prime} e^{-i t^{\prime}\left(\omega-p_{10}^{\prime}-p_{20}^{\prime}\right)} \int_{-\infty}^{t^{\prime}} d t e^{-i t\left(p_{10}+p_{20}-\omega\right)}\left[-i \gamma \cdot k \delta\left(k_{0}-\omega\right)\right]+\right. \\
\left.\int_{-\infty}^{\infty} d t e^{-i t\left(p_{10}+p_{20}+\omega\right)} \int_{-\infty}^{t} d t^{\prime} e^{i t^{\prime}\left(p_{10}^{\prime}+p_{20}^{\prime}+\omega\right)}\left[i \gamma \cdot k \delta\left(k_{0}+\omega\right)\right]\right\} \\
(2 \pi)^{3} \delta^{3}\left(\vec{p}_{1}+\vec{p}_{2}-\vec{p}_{1}^{\prime}-\vec{p}_{2}^{\prime}\right) \\
\equiv\left[S_{F}(k)+S_{B}(k)\right](2 \pi)^{4} \delta^{4}\left(p_{1}+p_{2}-p_{1}^{\prime}-p_{2}^{\prime}\right)
\end{gathered}
$$

where $\omega=\sqrt{k_{\perp}^{2}+\left(k_{z}\right)^{2}}$. We use light-cone coordinates, in which we define 


$$
\bar{n}^{\mu} \equiv\left(\bar{n}^{0}, \bar{n}_{\perp}, \bar{n}^{3}\right)=\frac{1}{\sqrt{2}}\left(1,0_{\perp}, 1\right) ; \quad n^{\mu}=\frac{1}{\sqrt{2}}\left(1,0_{\perp},-1\right)
$$

or equivalently

$$
\bar{n}^{\mu} \equiv\left(\bar{n}^{+}, \bar{n}^{-}, \bar{n}_{\perp}\right)=\left(1,0,0_{\perp}\right) ; \quad n^{\mu}=\left(0,1,0_{\perp}\right)
$$

Thus, $\bar{n}^{2}=0, n^{2}=0$, and $\bar{n} \cdot n=1 ; x=k \cdot n / P \cdot n$ is the light-cone fraction. In the collinear limit, we take $l_{\perp} \rightarrow 0$. In consequence, we have

$$
\begin{gathered}
\lim _{l_{\perp} \rightarrow 0} S_{F}(k)(2 \pi)^{4} \delta^{4}\left(p_{1}+p_{2}-p_{1}^{\prime}-p_{2}^{\prime}\right) \\
=\lim _{p_{10}+p_{20} \rightarrow \omega} \frac{\gamma \cdot k \delta\left(k_{0}-\omega\right)}{2 \omega} \int_{-\infty}^{\infty} d t^{\prime} e^{-i t^{\prime}\left(\omega-p_{10}^{\prime}-p_{20}^{\prime}\right)} \\
\left(\frac{e^{-i t^{\prime}\left(p_{10}+p_{20}-\omega\right)}}{p_{10}+p_{20}-\omega}-\frac{e^{-i T\left(p_{10}+p_{20}-\omega\right)}}{p_{10}+p_{20}-\omega}\right)(2 \pi)^{3} \delta^{3}\left(\vec{p}_{1}+\vec{p}_{2}-\vec{p}_{1}^{\prime}-\vec{p}_{2}^{\prime}\right) \\
=\frac{\sqrt{2} \omega \gamma \cdot \bar{n}}{2 \omega} \int_{-\infty}^{\infty} d t^{\prime} e^{-t^{\prime}\left(\omega-p_{10}^{\prime}-p_{20}^{\prime}\right)}\left(t^{\prime}-T\right)(2 \pi)^{3} \delta^{3}\left(\vec{p}_{1}+\vec{p}_{2}-\vec{p}_{1}^{\prime}-\vec{p}_{2}^{\prime}\right) \\
=\frac{\sqrt{2} \alpha \gamma \cdot \bar{n}}{2 \omega}(2 \pi)^{4} \delta^{4}\left(p_{1}+p_{2}-p_{1}^{\prime}-p_{2}^{\prime}\right) .
\end{gathered}
$$

Usually, the lower limit $\mathrm{T}$ of the integral, which is relating to the interaction time, is taken as $-\infty$. However, $\mathrm{T}$ is really a finite quantity in physics and we denote $t^{\prime}-T \equiv \alpha / \omega$, where $\alpha$ is a dimensionless quantity. We emphasis that the term $-e^{-i T\left(p_{10}+p_{20}-\omega\right)} /\left(p_{10}+\right.$ $\left.p_{20}-\omega\right)$ in Eq. (6), which vanishes in the noncollinear case, is important for the collinear safeness.

The second term in Eq. (3) is

$$
\lim _{l_{\perp} \rightarrow 0} S_{B}(k)(2 \pi)^{4} \delta^{4}\left(p_{1}+p_{2}-p_{1}^{\prime}-p_{2}^{\prime}\right)
$$




$$
\begin{gathered}
=\lim _{p_{10}^{\prime}+p_{20}^{\prime} \rightarrow \omega}-\frac{\gamma \cdot k \delta\left(k_{0}+\omega\right)}{2 \omega} \int_{-\infty}^{\infty} d t e^{-i t\left(\omega+p_{10}+p_{20}\right)} \frac{e^{i t\left(p_{10}^{\prime}+p_{20}^{\prime}+\omega\right)}}{p_{10}^{\prime}+p_{20}^{\prime}+\omega}(2 \pi)^{3} \delta^{3}\left(\vec{p}_{1}+\vec{p}_{2}-\vec{p}_{1}^{\prime}-\vec{p}_{2}^{\prime}\right) \\
=-\frac{\gamma \cdot k \delta\left(k_{0}+\omega\right)}{4 \omega^{2}}(2 \pi)^{4} \delta^{4}\left(p_{1}+p_{2}-p_{1}^{\prime}-p_{2}^{\prime}\right) \\
=\frac{\gamma \cdot n}{2 k \cdot n}(2 \pi)^{4} \delta^{4}\left(p_{1}+p_{2}-p_{1}^{\prime}-p_{2}^{\prime}\right)
\end{gathered}
$$

The negative frequency solution $k_{0}=-\omega$ in $S_{B}(k)$ can be understand as a backwardmoving parton in time order since $k \delta\left(k_{0}+\omega\right)=-\left(\omega, 0_{\perp},-k_{z}\right) \equiv \hat{k}, \hat{k}$ is on-shell momentum in TOPT. We will refer to $S_{F}(k)$ and $S_{B}(k)$ as the forward and backward propagators, respectively. We can generalize $\hat{k}$ to the off-shell momentum $\mathrm{k}$ in (6-7) since $n \cdot n=0$, where the transverse momentum of parton is vanished. Obviously, the backward propagator of quark is identical with the special propagator [4] or the instantaneous part of quark propagator in the non-collinear case [6]. Qiu has pointed out that the special propagator offers no space separation along the light-cone coordinate of two interaction points connected by the propagator. One can straightforwardly understand the above mentioned property as follows: the propagation of a backward parton "seems" to be instantaneous in kinematics when $P \rightarrow \infty$. It is interesting that Fig. $2 \mathrm{~b}$ shows that the virtual photon creates a $q \bar{q}$ pair in the infinite-momentum frame.

In general, the massless partons with the parallel momenta can go on-mass-shell simultaneously in the collinear case and collinear singularities may arise. Therefore, one might think that divergence could arise in the forward propagator $S_{F}(k)$ if $k^{2}=0$ (we call it as the collinear propagator). Fortunately, Eq. (6) shows that the collinear singularity is absent due to the compensative contributions from the factor $-e^{-i T\left(p_{10}+p_{20}-\omega\right)} /\left(p_{10}+p_{20}-\omega\right)$ and $\alpha / \omega$ is a finite quantity in Eq. (6). Furthermore, due to helicity conservation, the contributions of the numerator containing the collinear forward propagator to the twist-4 
structure functions vanish. For example, the emission of an on-shell massless collinear gluon along the quark line is forbidden in the collinear approximation. Therefore, one can neglect the collinear propagator $S_{F}(k)$ in the twist-4 component of structure function provided $\alpha / \omega$ is finite. Thus, we show a new way to eliminate collinear divergence: the collinear singularities will be transferred from the momentum-space to the interactiontime in TOPT and the later is really finite in physics.

Now let us discuss the gluon propagator $G^{\alpha \beta}(k)$. Similar to Eqs. (3-7), we have

$$
\begin{gathered}
\lim _{l_{\perp} \rightarrow 0} \int d^{4} k G^{\alpha \beta}(k) \\
=\frac{2 \alpha \Gamma^{\alpha \beta}(k) \delta\left(k_{0}-\omega\right)}{(k \cdot n)^{2}}(2 \pi)^{4} \delta^{4}\left(p_{1}+p_{2}-p_{1}^{\prime}-p_{2}^{\prime}\right)-\frac{\Gamma^{\alpha \beta}(k) \delta\left(k_{0}+\omega\right)}{(k \cdot n)^{2}}(2 \pi)^{4} \delta^{4}\left(p_{1}+p_{2}-p_{1}^{\prime}-p_{2}^{\prime}\right) \\
\equiv\left[G_{F}^{\alpha \beta}(k)+G_{B}^{\alpha \beta}(k)\right](2 \pi)^{4} \delta^{4}\left(p_{1}+p_{2}-p_{1}^{\prime}-p_{2}^{\prime}\right)
\end{gathered}
$$

where $\Gamma^{\alpha \beta}$ is the gauge-dependent polarization sum. In the light-cone gauge and limit $l_{\perp} \rightarrow 0$

$$
\begin{gathered}
\Gamma^{\alpha \beta}(k) \delta\left(k_{0}-\omega\right)=-g^{\alpha \beta}+\frac{k^{\alpha} n^{\beta}+k^{\beta} n^{\alpha}}{2 \omega} \delta\left(k_{0}-\omega\right) \\
=\delta_{\perp}^{i j},
\end{gathered}
$$

in $G_{F}^{\alpha \beta}(k)$

and

$$
\begin{aligned}
-\Gamma^{\alpha \beta}(k) \delta\left(k_{0}+\omega\right) & =g^{\alpha \beta}-\frac{k^{\alpha} n^{\beta}+k^{\beta} n^{\alpha}}{2 \omega} \delta\left(k_{0}+\omega\right) \\
& =g^{\alpha \beta}+n^{\alpha} n^{\beta}
\end{aligned}
$$

in $G_{B}^{\alpha \beta}(k)$, respectively. Where we use $\omega=x p$. Therefore, 


$$
G_{F}^{\alpha \beta}(k)=\frac{2 \alpha \delta_{\perp}^{i j}}{(k \cdot n)^{2}}
$$

and

$$
G_{B}^{\alpha \beta}(k)=\frac{g^{\alpha \beta}}{(k \cdot n)^{2}}+\frac{n^{\alpha} n^{\beta}}{(k \cdot n)^{2}}
$$

where $\delta_{\perp}^{i j}$ and $g^{\alpha \beta}$ collect the contributions of the terms with $l_{\perp}^{2}$ in the numerator. Thus, in the collinear limit, we need only consider the contributions of

$$
G_{B}^{\alpha \beta}(k)=\frac{n^{\alpha} n^{\beta}}{(k \cdot n)^{2}}
$$

in calculations of the twist-4 structure functions. Equation (13) is the special propagator $G_{s}^{\alpha \beta}(k)[4]$ or the instantaneous propagator for gluon in the noncollinear case [6].

From the above discussions, we can conclude that the contributions of the vertices (Figs. 3a and 3b) vanish due to collinear safeness, where $\mathrm{F}$ and $\mathrm{B}$ label the forward- and backward-moving partons, respectively. Furthermore, the vertices in Figs. 3c and 3d also are forbidden due to $\gamma \cdot n \gamma \cdot n=0$ and $n \cdot n=0$.

The amplitude with twist-4 corrections in the collinear expansion can contain the noncollinear propagators with off-shell momenta although parton transverse momenta are neglected. Any noncollinear propagators can be decomposed to a non-vanishing forward and backward components in TOPT, in which $S_{B}(k)$ and $G_{B}^{\alpha \beta}(k)$ have the same form as (7) and (13), respectively. On the other hand, we can use stand TOPT to calculate $S_{F}(k)$ and $G_{F}(k)$ and get

$$
S_{F}(k)=\frac{\gamma \cdot \bar{n}}{2 k \cdot \bar{n}}
$$


and

$$
G_{F}^{\alpha \beta}(k)=\frac{\delta_{\perp}^{i j}}{k \cdot \bar{n}(k \cdot n-k \cdot \bar{n})},
$$

where $\mathrm{k}$ are off-shell momenta. From the discussions about the propagators, we can find that the backward-propagating gluon is longitudinally polarized, while the forwardpropagating gluon is transversely polarized in the collinear approximation. A parton will inverse (or will keep) its moving direction after absolving a forward (or backward) gluon due to helicity conservation (Fig. 4). It means that the vertices of Figs. 3e-g are forbidden. Thus, a backward-moving gluon only couples with two forward-moving partons, while a forward-moving gluon couples with a pair of quark and antiquark moving oppositely.

The selecting rules (Fig. 4) simplify the calculations of the twist-4 corrections in the collinear expansion. In fact, we have the following TOPT-procedure for calculating the high-twist contributions in the collinear expansion: (1) to list all possible time-ordered diagrams of a photon-parton forward scattering amplitudes; (2) to label B or F on the parton lines according to Fig. 4, and note that all loop momenta are forward while the cut propagator is denoted by $\mathrm{F}$ or $\mathrm{B}$ for gluon or fermion, respectively, since the polarization sums of initial and final gluons have the same form $\delta_{\perp}^{i j} ;(3)$ the contributions of the backward and noncollinear forward propagators are calculated by $S_{B}(k), G_{B}^{\alpha \beta}(k)$, $S_{F}(k)$ and $G_{F}^{\alpha \beta}(k)$, respectively. One can find that every cut-diagram only corresponds to a non-vanished TOPT-graph due to the coupling of partons in space-time (Fig. 4). In opposition to our TOPT-method, the covariant perturbative methods $[3,4]$ do not tell whether forward propagator or backward propagator is more important, since they mix together all possible time ordering in Feynman diagram. Obviously, the gauge invariance 
is preserved if we include all TOPT-diagrams of a physical process at given order of coupling constant.

\section{Power corrections to structure functions}

One can simply reproduce all results of Ref. [4] using our TOPT-programs in Sec. 2. In deep inelastic scattering of lepton-hadron, the hadronic tensor $W^{\mu \nu}(p, q)$ can decompose to

$$
W^{\mu \nu}(p, q)=e_{L}^{\mu \nu}\left(x_{B}, Q^{2}\right)+e_{T}^{\mu \nu}\left(x_{B}, Q^{2}\right)
$$

where $e_{L}^{\mu \nu}$ and $e_{T}^{\mu \nu}$ are the longitudinal and transverse tensors, respectively. Using the definition (4),

$$
\begin{gathered}
e_{L}^{\mu \nu}=\frac{1}{2}\left(g^{\mu \nu}+\frac{\left(x_{B} p\right)^{2}}{Q^{2}} \bar{n}^{\mu} \bar{n}^{\nu}+\frac{Q^{2}}{\left(2 x_{B} p\right)^{2}} n^{\mu} n^{\nu}-\frac{1}{2}\left(\bar{n}^{\mu} \bar{n}^{\nu}\right)\right), \\
e_{T}^{\mu \nu}=\frac{1}{2}\left(\bar{n}^{\mu} n^{\nu}+n^{\mu} \bar{n}^{\nu}-g^{\mu \nu}\right)=\frac{1}{2} d^{\mu \nu} .
\end{gathered}
$$

The standard structure functions are defined as

$$
\begin{gathered}
2 F_{1}\left(x_{B}, Q^{2}\right)=F_{T}\left(x_{B}, Q^{2}\right), \\
F_{2}\left(x_{B}, Q^{2}\right)=x_{B}\left[F_{T}\left(x_{B}, Q^{2}\right)+F_{L}\left(x_{B}, Q^{2}\right)\right] .
\end{gathered}
$$

The inverse-power corrections of the twist-4 QCD processes to the structure functions $F_{1}$ and $F_{2}$, or the hadronic tensor $W^{\mu \nu}$ are from Fig. 5 (for quark-gluon-to-quark-gluon) 
and Fig. 6 (for two-quark-to-two-quark), respectively. In the concrete, $W_{4}^{\mu \nu} \sim \int \sigma^{\mu \nu} T$, where $\sigma^{\mu \nu}$ are the cross section of the processes in Figs. 5 and 6 , $\mathrm{T}$ is the contributions from the target part $[4,7]$. Using our calculating program in TOPT, we can easy write $\sigma^{\mu \nu}$ in Fig. 5 as

$$
\begin{aligned}
& \sigma_{q g a}^{\mu \nu}\left(x, x_{1}, x_{2}\right) \\
& =\frac{d^{\alpha \beta}}{16 \pi} \operatorname{Tr}\left[\gamma^{\alpha} \frac{\gamma \cdot n}{2 k \cdot n} \gamma^{\mu} \frac{Q^{2}}{2 x_{B} p} \gamma \cdot n \gamma^{\nu} \frac{\gamma \cdot n}{2 k \cdot n} \gamma^{\beta} p \gamma \cdot \bar{n}\right] 2 \pi \delta\left(x-x_{B}\right) \frac{x_{B}}{Q^{2}} \\
& =2 n^{\mu} n^{\nu} \delta\left(x-x_{B}\right)\left(\frac{1}{2 x p}\right)^{2}, \\
& \sigma_{q g b}^{\mu \nu}\left(x, x_{1}, x_{2}\right) \\
& =\frac{d^{\alpha \beta}}{16 \pi} \operatorname{Tr}\left[\gamma^{\mu} \frac{x_{B} p}{Q^{2}} \gamma \cdot \bar{n} \gamma^{\alpha} \frac{Q^{2}}{2 x_{B} p} \gamma \cdot n \gamma^{\beta} \frac{x_{B} p}{Q^{2}} \gamma \cdot \bar{n} \gamma^{\nu} p \gamma \cdot \bar{n}\right] 2 \pi \delta\left(x-x_{B}\right) \frac{x_{B}}{Q^{2}} \\
& =2 \bar{n}^{\mu} \bar{n}^{\nu} \delta\left(x-x_{B}\right)\left(\frac{p x}{Q^{2}}\right)^{2}, \\
& \sigma_{q g c}^{\mu \nu}\left(x, x_{1}, x_{2}\right) \\
& =\frac{d^{\alpha \beta}}{16 \pi} \operatorname{Tr}\left[\gamma^{\mu} \frac{Q^{2}}{2 x_{B} p} \gamma \cdot n \gamma^{\alpha} \frac{x_{B} p}{Q^{2}} \gamma \cdot \bar{n} \gamma^{\beta} \frac{\gamma \cdot n}{2\left(x_{1}-x_{B}\right) x p} \gamma^{\nu} p \gamma \cdot \bar{n}\right] 2 \pi \delta\left(x_{2}-x_{B}\right) \frac{x_{B}}{Q^{2}} \\
& =e_{T}^{\mu \nu} \delta\left(x_{2}-x_{B}\right) \frac{x_{2}}{\left(x_{1}-x_{2}\right) Q^{2}}, \\
& \sigma_{q g e}^{\mu \nu}\left(x, x_{1}, x_{2}\right) \\
& =\frac{d^{\alpha \beta}}{16 \pi} \operatorname{Tr}\left[\gamma^{\mu} \frac{x_{B} p}{Q^{2}} \gamma \cdot \bar{n} \gamma^{\alpha} \frac{Q^{2}}{2 x_{B} p} \gamma \cdot n \gamma^{\nu} \frac{\gamma \cdot n}{2 x p} \gamma^{\beta} p \gamma \cdot \bar{n}\right] 2 \pi \delta\left(x-x_{B}\right) \frac{x_{B}}{Q^{2}} \\
& =\bar{n}^{\mu} n^{\nu} \delta\left(x-x_{B}\right) \frac{1}{Q^{2}} \text {. }
\end{aligned}
$$


Note that the contributions of Figs.5g-5j are vanished since in Figs. $5 \mathrm{~g}$ and $5 \mathrm{~h}$ the propagators $\mathrm{F}_{1}$ contributes $\sim \gamma \cdot \bar{n} / p$ and leads to $\sigma_{T}^{\mu \nu} / p^{2} \rightarrow 0$ if $p \rightarrow \infty$; Figs. 5i and 5j include $\operatorname{Tr}\left[\gamma^{\mu} \gamma \cdot n \gamma^{\alpha} \gamma \cdot \bar{n} \gamma^{\nu} \gamma \cdot n \gamma^{\beta} \gamma \cdot \bar{n}\right]=0$

Similar, the contributions of four-quark process to the leading inverse power corrections in Fig. 6 are

$$
\begin{gathered}
\sigma_{q q a}^{\mu \nu}\left(x, x_{1}, x_{2}\right) \\
=\frac{d^{\alpha \beta}}{16 \pi} \operatorname{Tr}\left[\gamma^{\mu} \frac{\gamma \cdot n}{2\left(x_{2}-x_{B}\right) p} \gamma^{\alpha} p \gamma \cdot \bar{n} \gamma^{\beta} \frac{\gamma \cdot n}{2\left(x_{1}-x_{B}\right) p} \gamma^{\nu} p \gamma \cdot \bar{n}\right] 2 \pi \delta\left(x-x_{B}\right) \frac{x_{B}}{Q^{2}} \\
=e_{T}^{\mu \nu} \delta\left(x-x_{B}\right) \frac{x}{\left(x_{1}-x\right)\left(x_{2}-x\right) Q^{2}}, \\
=\frac{d^{\alpha \beta}}{16 \pi} \operatorname{Tr}\left[\gamma^{\mu} \frac{\sigma_{q q b}^{\mu \nu}\left(x, x_{1}, x_{2}\right)}{2\left(x_{2}-x_{B}\right) p} \gamma^{\alpha} p \gamma \cdot \bar{n} \gamma^{\beta} \frac{Q^{2}}{2\left(x_{2}-x_{B}\right) p} \gamma^{\alpha} p \gamma \cdot \bar{n}\right] \frac{2 x_{1}}{\left(x-x_{1}\right) Q^{2}} 2 \pi \delta\left(x_{1}-x_{B}\right) \frac{x_{B}}{Q^{2}} \\
=e_{T}^{\mu \nu} \delta\left(x_{1}-x_{B}\right) \frac{x_{1}}{\left(x-x_{1}\right)\left(x_{2}-x_{1}\right) Q^{2}} .
\end{gathered}
$$

Thus, we reproduce the results of Refs. [3,4] using TOPT. They are

$$
\begin{gathered}
\sigma_{q g}^{\mu \nu}\left(x, x_{1}, x_{2}\right) \\
=\frac{8}{Q^{2}}\left(e_{T}^{\mu \nu}+e_{L}^{\mu \nu}\right) \delta\left(x-x_{B}\right)-\frac{2 x_{B}}{Q^{2}} e_{T}^{\mu \nu}\left[\frac{\delta\left(x_{2}-x_{B}\right)-\delta\left(x_{1}-x_{B}\right)}{x_{2}-x_{1}}\right], \\
=\frac{x_{B}^{\mu \nu}\left(x, x_{1}, x_{2}\right)}{Q^{2}} e_{T}^{\mu \nu}\left[\frac{\delta\left(x_{2}-x_{B}\right)-\delta\left(x_{1}-x_{B}\right)}{\left(x_{2}-x\right)\left(x_{2}-x_{1}\right)}-\frac{\delta\left(x-x_{B}\right)-\delta\left(x_{1}-x_{B}\right)}{\left(x_{2}-x\right)\left(x-x_{1}\right)}\right. \\
\left.+\frac{\delta\left(y_{2}-x_{B}\right)-\delta\left(y_{1}-x_{B}\right)}{\left(y_{2}-x\right)\left(y_{2}-y_{1}\right)}-\frac{\delta\left(y-x_{B}\right)-\delta\left(y_{1}-x_{B}\right)}{\left(y_{2}-x\right)\left(x-y_{1}\right)}\right]
\end{gathered}
$$


where $y_{1}=x_{1}-x$ and $y_{2}=x-x_{2}$.

In the above mentioned calculations we considered the processes of two-parton-to-twoparton. We also should pick up the contributions from all cut diagrams of the interference process of one-parton-to-one-parton and three-parton-to-one-parton. For example, we need include contributions of Fig. 7, which are corresponding to Fig. 5c. One can find that the contributions of Figs. 7a and 7b are the same as that of Fig. 5c. Therefore, we should multiply the above mentioned results (26) and (27) by a factor 3 for including the contributions of the interference processes.

\section{Factorization scheme in TOPT}

A relating subject with the above mentioned statements of power corrections to structure functions is the factorization scheme in TOPT. As we know that factorization theorem allows us to separate the calculable short-distance effects from incalculable long-distance part. A complete derivation of the factorization theorem in DIS has been given by many authors [8]. The discussion of this section intends to provide an intuitive understanding of the existing factorization scheme.

A simplest amplitude of DIS process contains a pair of (left and right) loop Feynman propagators and it can be written as

$$
M^{\mu \nu}(p, q)=\operatorname{Tr}\left[\gamma \cdot p T D H N H^{*} D^{*} T^{*}\right],
$$

where $D$ and $D^{*}$ are Feynman propagators of quarks connecting with the target-part $T$ $\left(T^{*}\right)$ and hard-part $H\left(H^{*}\right)$, respectively. According to the view of parton model, in a factorization scheme, all virtual (off-shell) partonic lines joining $T$ and $H$ can be broken 
to the free (on-shell) partonic lines. In this case, we can re-stipulate those on-shell partons as incoming and outgoing partons in the target- and hard-parts, respectively.

Any Feynman propagator is decomposed to the forward $F$ and backward $B$ propagators and the propagating partons are evaluated on-shell in TOPT. The diagram including two forward propagators in Fig. 8a gives the leading twist-2 contribution and we can separate the calculable short-distance effects from incalculable target part, since two onshell-forward propagators can be broken to incoming and outgoing partons in the target and hard parts in a factorization scheme, i.e.,

$$
\begin{gathered}
M^{\mu \nu}(p, q)=\operatorname{Tr}\left[\gamma \cdot p T \gamma \cdot \hat{k} H N H^{*} \gamma \cdot \hat{k} T^{*}\right] \\
=\operatorname{Tr}\left[T^{*} \gamma \cdot p \operatorname{T\gamma } \cdot \hat{k}\right] \operatorname{Tr}\left[H N H^{*} \gamma \cdot \hat{k}\right],
\end{gathered}
$$

where $\hat{k}$ is on-shell momentum of the propagator $F$. The first factor in Eq. (29) is the trace of the (soft) forward virtual quark-target scattering amplitude, and the second factor relates to the (hard) probe-parton subprocess.

On the other hand, the backward propagator implies the contact (or instantaneous) correlation and we can not re-stipulate the backward propagator in any factorization scheme. Therefore, one of the factorization conditions is that we can avoid the backward propagators between $T$ and $H$. For this end, we note that a backward parton always couples with a pair of forward partons according to Fig. 4. After "pulling down" two forward propagators from the target matrix, the backward propagator can be isolated into a hard-subgraph of a next twist amplitude. In consequence, we get the twist-3 (Figs. 8b and 8c) and twist-4 contributions (Fig. 8d), respectively. In this case, all initial partons are forward moving along the direction of target and we can break and recombine those 
parton lines between two factorized parts in the factorization scheme. Through the above TOPT-expansion, we also show a clear picture where the different twist contributions are separated.

\section{Summary}

In this work, we presented following physical picture about the inverse-power corrections to the hadron structure in parton model: (i) a hadron is assumed to be consisted by forward initial partons along the target momentum in the infinite momentum frame (i.e., the positively of initial parton momentum) and the transverse momentum of parton can be neglected if comparing with its longitudinal momentum; (ii) a probe strikes with a parton and forms a covariant Feynman propagator connecting with the target matrix and probe; (iii) the Feynaman propagator decomposes to the forward and backward propagators in TOPT and the backward propagator "pulls down" two forward propagators from the target matrix; (iv) after factorization, two-parton-correlation function has the dimension of $[\text { length }]^{-2}$, therefore, the hard-amplitude of two-parton-to-two-parton will provide a contribution with $1 / Q^{2}$ to the total amplitude. This leads an inverse power correction to the hadron structure function.

In summary, we have generalized time-ordered perturbative theory to the collinear limit, where the collinear singularities are eliminated in a natural way. We find that a clear space-time picture of the twist-4 amplitude is presented in its TOPT-form. In particular, we identify the special propagators with the backward propagators of partons. Our method provides a new way to analyze the twist-4 processes in a consistent QCDimproved parton model. 
Acknowledgments: We would like to thank J.W. Qiu for his encouragement and enlightening discussions. We would also like to acknowledge D. Indumathi for useful comments. This work was supported by National Natural Science Foundation of China and '95-climbing' Plan of China. 


\section{References}

[1] A. De Rujula, H. Georgi and H.D. Polotzer, Ann. Phys. (N.Y.) 103, 315 (1977); H.D. Politzer, Nucl. Phys. B172, 349 (1980); S.J. Brodsky, E. L. Berger and G.P. Lepage, in Proceedings of the Workshop on Drell-Yan Processes, Batavia, Illinois, 1982 (Fermilab, Batavia, 1983), p. 137.

[2] R.L. Jaffe and M. Soldate, Phys. Lett. 105B (1981) 467; Phys. Rev. D26, 49 (1982).

[3] R.K. Ellis, W. Furmanski and R. Petronzio, Nucl. Phys. B207, 1 (1982); B212, 29 (1983).

[4] J.W. Qiu, Phys. Rev. D42, 30 (1990).

[5] G. Altarelli and G. Parisi, Nucl. Phys. B126, 298 (1977).

[6] S.J. Brodsky, Lecture at the Summer Institute on Particle Physics at SLAC, California, 1979 .

[7] X.F. Guo and J.W. Qiu, The leading power corrections to the structure functions, BNL/HET-98/36.

[8] J.C. Collins, D.E. Soper and G. Sterman, in Perturbative Quantum Chromodynamics, edited by A.H. Mueller (World Scientific, Singapore) (1989) 1; J.C. Collins and D.E. Soper, Nucl. Phy. B194 (1982) 445. 


\section{Figure Captions}

Fig. 1 A twist-2 photon-hadron scattering process of $\mathcal{O}\left(\alpha_{s}\right)$ in TOPT; (a) dominant amplitude with the forward propagator and (b) suppressed amplitude with the backward propagator.

Fig. 2 A twist-4 photon-hadron scattering process of $\mathcal{O}\left(\alpha_{s}\right)$ in TOPT; (a) suppressed amplitude with the forward propagator and (b) dominant amplitude with the backward propagator.

Fig. 3 Some forbidden vertices of partons in twist-4 photon-hadron scattering amplitude. $\mathrm{F}$ and $\mathrm{B}$ indicate forward- and backward-moving partons, respectively.

Fig. 4 Elemental vertices in the collinear approximation.

Fig. 5 A complete set of quark-gluon-to-quark-gluon TOPT diagrams, which give leading power corrections to the hadron structure function.

Fig. 6 Non-vanished two-quark-to-two-quark TOPT diagrams, which give leading power corrections to the hadron structure function.

Fig. 7 Interference processes corresponding to Fig. 5c.

Fig. 8 TOPT-expansion of the different twist contributions. 

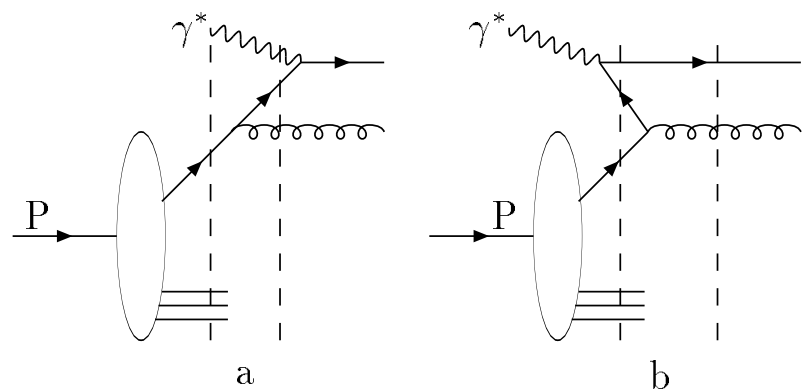

Fig.1 


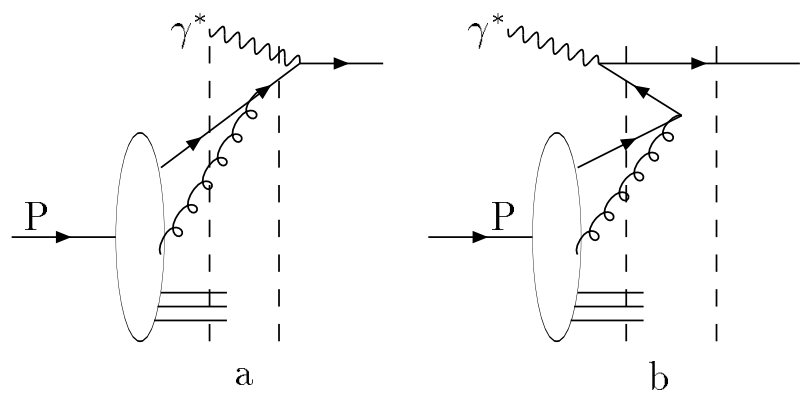

Fig.2 


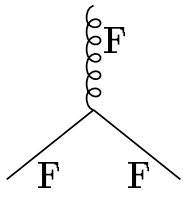

(a)

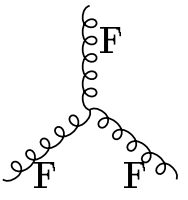

(b)

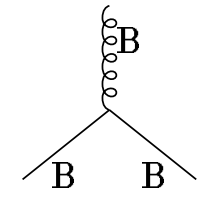

(c)

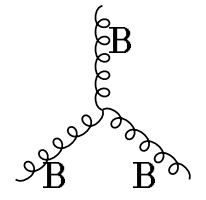

(d)<smiles>CB=C(C)C</smiles>

(e)<smiles>[B]C(C)[B]C</smiles>

(f)<smiles>[10BH]C(=P)PC</smiles>

(g)

Fig.3 


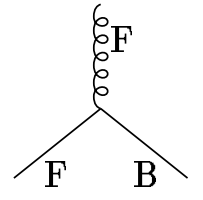

(a)

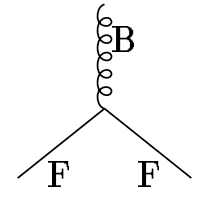

(b)

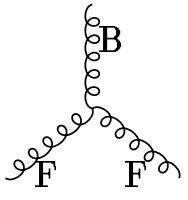

(c)

Fig.4 


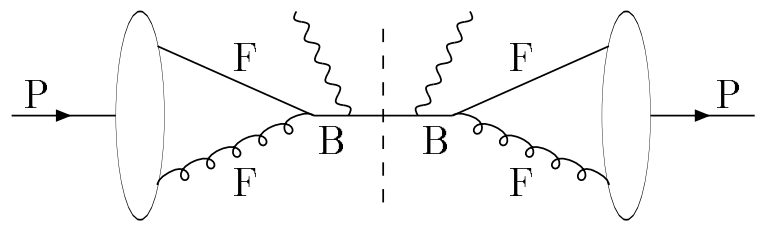

(a)

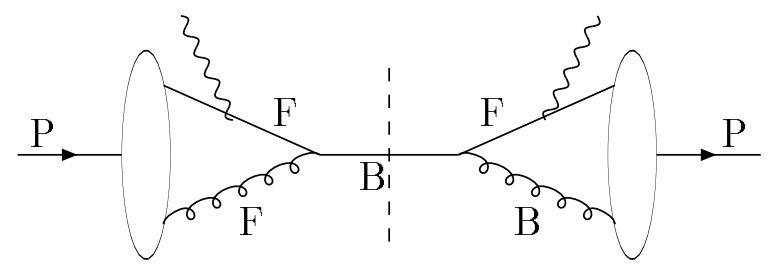

(b)

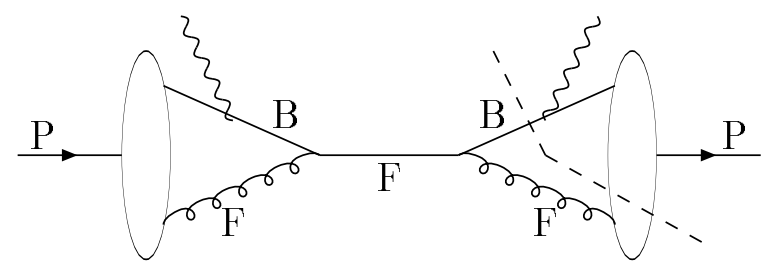

(c)

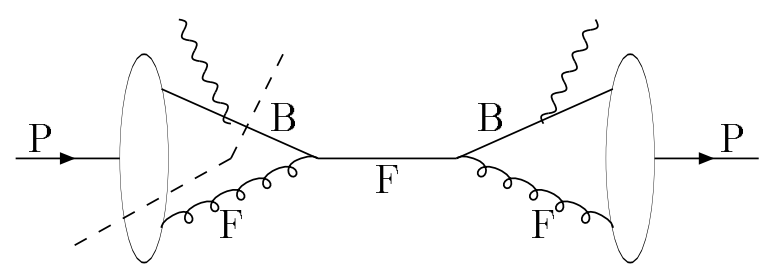

(d) 


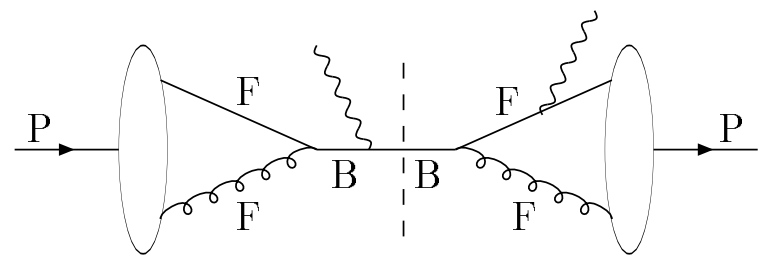

(e)

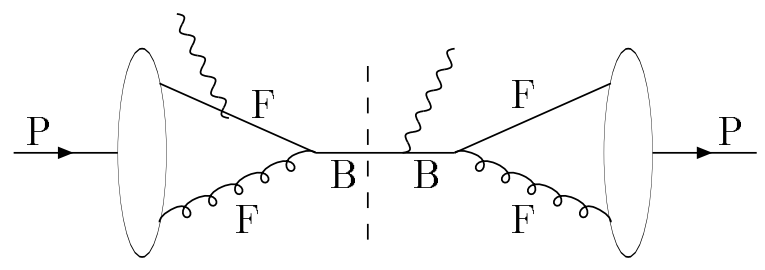

(f)

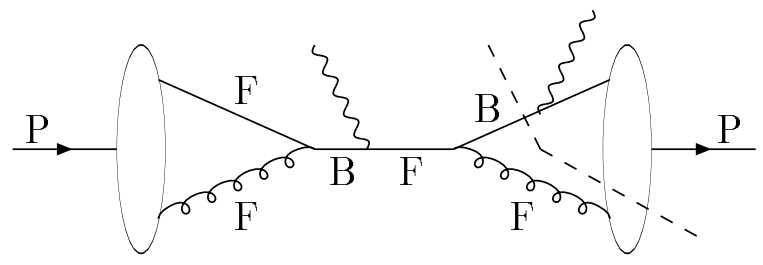

(g)

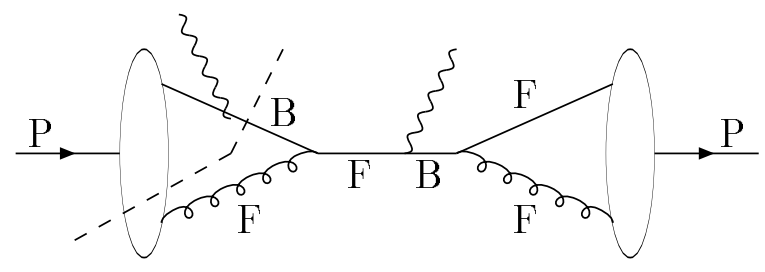

(h)

2 


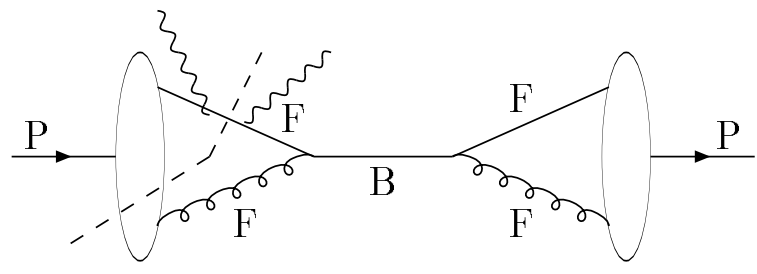

(i)

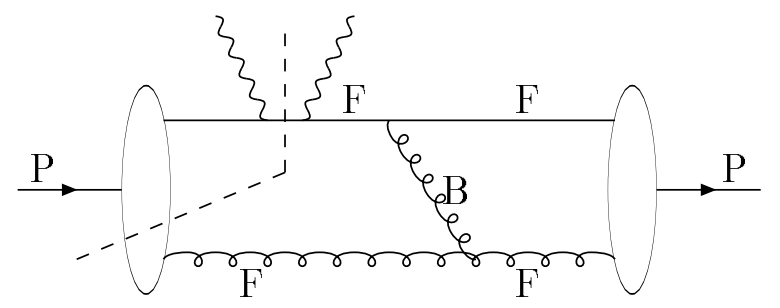

(i)

Fig.5 


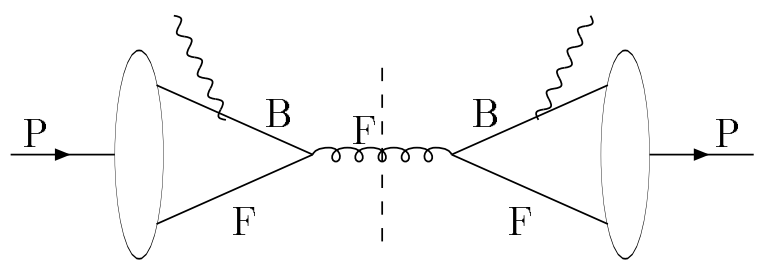

(a)

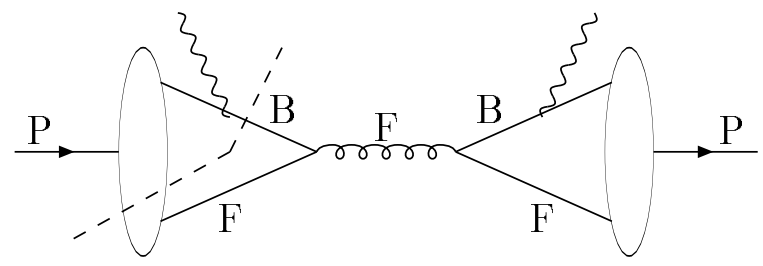

(b)

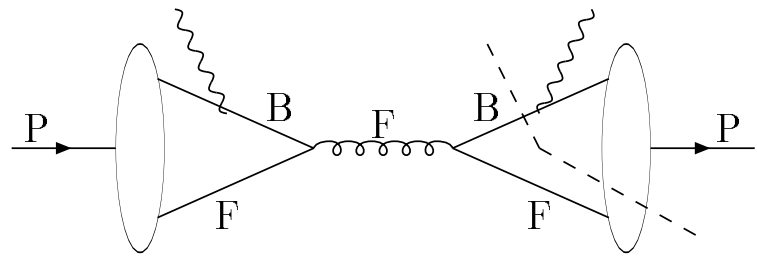

(c)

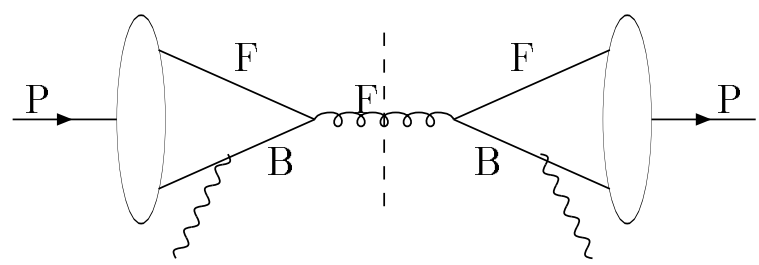

(d) 


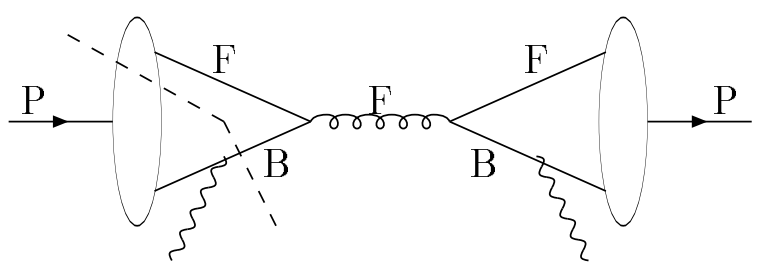

(e)

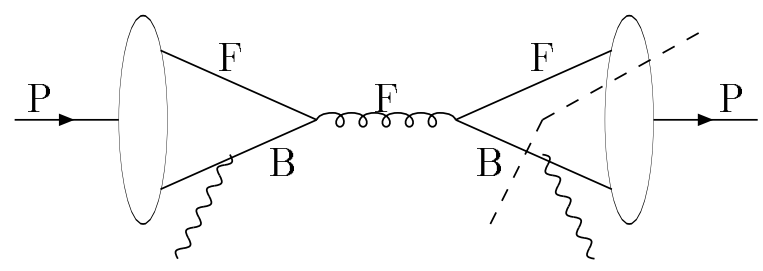

(f)

Fig.6 


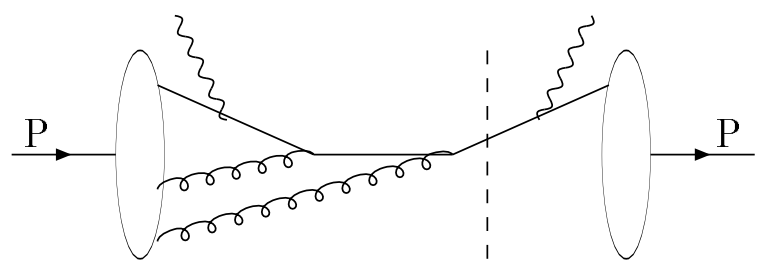

(a)

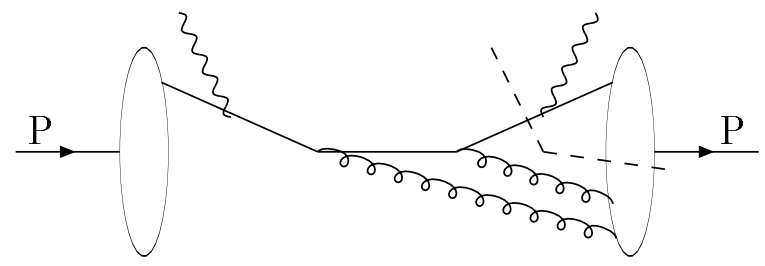

(b)

Fig.7 


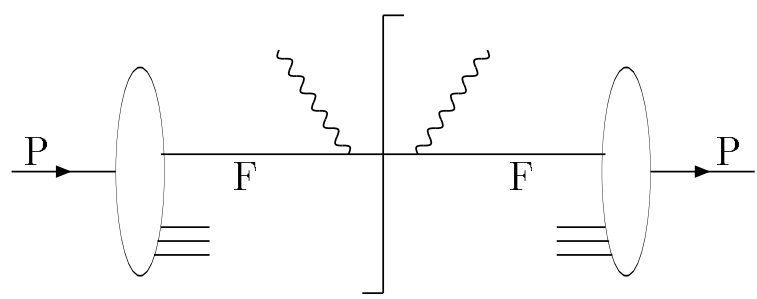

(a)

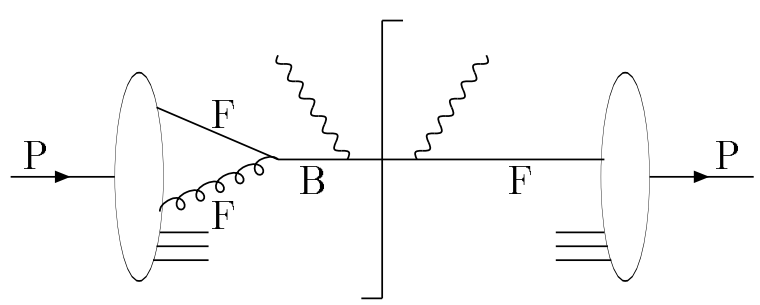

(b)

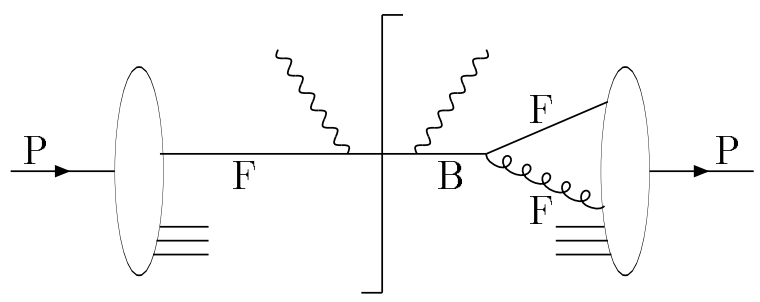

(c)

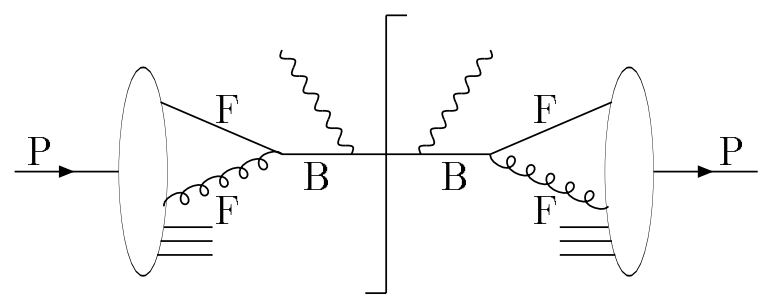

(d)

Fig.8 